Sixty patients with a history of deep vein thrombosis were prospectively studied. All were judged likely to have residual ultrasound abnormalities but had no symptoms of recurrence. Two experienced ultrasonographers, blinded to the locations of the original thrombi, examined the proximal deep veins of both legs of each patient. The lengths of any incompressible segments were measured using four anatomical landmarks as reference points, and results from each sonographer were compared.

The results showed good interobserver agreement on thrombus length, although measurements using the inguinal ligament as a landmark were less precise. The authors conclude that an apparent increase of $\geq 9 \mathrm{~cm}$ is likely to indicate thrombus extension when measured in this standardized way. Smaller increases, however, are likely to be attributable to measurement error. They note that their findings are preliminary and require confirmation in a prospective clinical trial.

Original article Linkins L-A et al. (2004) Change in thrombus length on venous ultrasound and recurrent deep vein thrombosis. Arch Intern Med 164: 1793-1796

\section{Influence of renal impairment on cardiovascular risk}

Following myocardial infarction, the risk of further cardiovascular events is increased by the presence of renal failure. It is not clear whether milder renal impairment is also a significant risk factor. Anavekar et al. have investigated this role as part of the Valsartan in Acute Myocardial Infarction Trial (VALIANT).

The study randomized 14,527 high-risk survivors of acute myocardial infarction to receive captopril, valsartan or both, in a 1:1:1 ratio. As inhibitors of the renin-angiotensin system, these drugs are nephroprotective and reduce cardiovascular risk. The patients were categorized according to their baseline renal function-as indicated by the glomerular filtration rate (GFR)-and comparisons were made between these groups.

Baseline GFR measurements suggested that approximately a third of the patients had chronic kidney disease. Those in the lowest GFR category $\left(<45.0 \mathrm{ml}\right.$ per minute per $\left.1.73 \mathrm{~m}^{2}\right)$ had the highest prevalence of coexisting risk factors. These patients were the least likely to have received other cardiovascular pharmacotherapies or to have undergone coronary revascularization. Mortality and cardiovascular risk were higher in patients with lower estimated GFR values at baseline: below $81.0 \mathrm{ml}$ per minute per $1.73 \mathrm{~m}^{2}$, each 10 -unit reduction in GFR brought a $10 \%$ increase in the relative risk of death or nonfatal cardiovascular complications. This was the case in all treatment groups.

Anavekar et al. conclude that any degree of renal disease should be viewed as a significant independent risk factor in patients with myocardial infarction.

Original article Anavekar NS et al. (2004) Relation between renal dysfunction and cardiovascular outcomes after myocardical infarction. N Engl J Med 351: 1285-1295

\section{Invasive versus conservative strategy for STEMI}

Current guidelines discourage early postthrombolysis routine angioplasty in patients with ST-segment elevated myocardial infarction (STEMI). Since the advent of stents and new antiplatelet agents, however, this advice may no longer be appropriate. The multicenter GRACIA-1 trial compared a routine invasive strategy with a conservative approach.

Following thrombolysis for STEMI, 500 patients were randomly assigned to undergo angiography and revascularization within $24 \mathrm{~h}$ if indicated ( $n=248)$, or to an ischemia-guided, conservative strategy $(n=252)$. The majority $(80 \%)$ of patients in the invasive treatment group underwent stenting of the culprit artery, whereas $1 \%$ underwent nonculprit-artery stenting and $2 \%$ received coronary bypass surgery. No revascularization treatment was indicated in the remaining $17 \%$ of patients in this group. In the conservative group, ischemia-guided revascularization was carried out in $20 \%$ of patients before discharge.

The need for unplanned revascularization was significantly lower among patients in the invasive group, than among those treated conservatively. Although the trial was not powered to detect differences in the rates of death or re-infarction, the composite endpoint of death, re-infarction or revascularization at 1 year was also significantly less frequent in the invasive group. 